\title{
The Dual Role of Lipids of the Lipoproteins in Trumenba, a Self-Adjuvanting Vaccine Against Meningococcal Meningitis B Disease
}

\author{
Yin Luo, ${ }^{1,6}$ Olga V. Friese, ${ }^{2}$ Herbert A. Runnels, ${ }^{2}$ Lakshmi Khandke, ${ }^{3}$ Gary Zlotnick, ${ }^{3}$ Ann Aulabaugh, ${ }^{4}$ \\ Thomas Gore, ${ }^{5}$ Eugene Vidunas, ${ }^{3}$ Stephen W. Raso, ${ }^{1}$ Elena Novikova, ${ }^{3}$ Emilia Byrne, ${ }^{2}$ Michael Schlittler, ${ }^{2}$ \\ Donald Stano, ${ }^{2}$ Robert L. Dufield, ${ }^{1}$ Sandeep Kumar, ${ }^{2}$ Annaliesa S. Anderson, ${ }^{3}$ \\ Kathrin U Jansen, ${ }^{3}$ and Jason C. Rouse ${ }^{1,6}$
}

Received 30 June 2016; accepted 17 August 2016; published online 7 September 2016

\begin{abstract}
Trumenba (bivalent rLP2086) is a vaccine licensed for the prevention of meningococcal meningitis disease caused by Neisseria meningitidis serogroup B $(\mathrm{NmB})$ in individuals 10-25 years of age in the USA. The vaccine is composed of two factor $\mathrm{H}$ binding protein (fHbp) variants that were recombinantly expressed in Escherichia coli as native lipoproteins: rLP2086-A05 and rLP2086-B01. The vaccine was shown to induce potent bactericidal antibodies against a broad range of $\mathrm{NmB}$ isolates expressing $\mathrm{fHbp}$ that were different in sequence from the fHbp vaccine antigens. Here, we describe the characterization of the vaccine antigens including the elucidation of their structure which is characterized by two distinct motifs, the polypeptide domain and the N-terminal lipid moiety. In the vaccine formulation, the lipoproteins self-associate to form micelles driven by the hydrophobicity of the lipids and limited by the size of the folded polypeptides. The micelles help to increase the structural stability of the lipoproteins in the absence of bacterial cell walls. Analysis of the lipoproteins in Toll-like receptor (TLR) activation assays revealed their TLR2 agonist activity. This activity was lost with removal of the $O$-linked fatty acids, similar to removal of all lipids, demonstrating that this moiety plays an adjuvant role in immune activation. The thorough understanding of the structure and function of each moiety of the lipoproteins, as well as their relationship, lays the foundation for identifying critical parameters to guide vaccine development and manufacture.
\end{abstract}

KEY WORDS: bivalent rLP2086; factor $\mathrm{H}$ binding protein; meningococcal meningitis $\mathrm{B}$ vaccine; Neisseria meningitidis serogroup B; self-adjuvanting vaccine.

Electronic supplementary material The online version of this article (doi:10.1208/s12248-016-9979-x) contains supplementary material, which is available to authorized users.

${ }^{1}$ Pfizer Biotherapeutics Pharmaceutical Sciences, 1 Burtt Rd., Andover, Massachusetts, USA.

${ }^{2}$ Pfizer Biotherapeutics Pharmaceutical Sciences, 700 Chesterfield Pkwy W, St. Louis, Missouri, USA.

${ }^{3}$ Pfizer Vaccine Research and Development, 401 N. Middletown Rd., Pearl River, New York, USA.

${ }^{4}$ Pfizer Worldwide Research, 558 Eastern Point Rd., Groton, Connecticut, USA.

${ }^{5}$ Pfizer Biotherapeutics Pharmaceutical Sciences, 401 N. Middletown Rd., Pearl River, New York, USA.

${ }^{6}$ To whom correspondence should be addressed. (e-mail: yin.luo@pfizer.com; jason.rouse@pfizer.com)

ABBREVIATIONS NmB, Neisseria meningitidis serogroup $\mathrm{B}$; fHbp, Factor H binding protein; TLR, Toll-like receptor; GC/MS, Gas chromatography-mass spectrometry; LC/MS, Liquid chromatography-mass spectrometry; DSC, Differential scanning calorimetry; MALS, Multi-angle light scattering; AUC, Analytical ultracentrifugation; CMC, Critical micellar concentration; PS-80, Polysorbate 80; SBA, Serum bactericidal antibody assay

\section{INTRODUCTION}

Neisseria meningitidis, also called meningococcus (Men), is a human commensal Gram-negative bacterium that can cause severe disease with substantial mortality and morbidity. Survivors can suffer from permanent neurological sequelae and limb loss. $N$. meningitidis is categorized by its capsular polysaccharides into 12 serogroups, of which six cause the majority of disease (1). Four of the serogroups (A, $\mathrm{C}, \mathrm{Y}, \mathrm{W})$ can be controlled with capsular polysaccharidebased vaccines. A capsular polysaccharide-based vaccine approach was not feasible for protection against $N$. meningitidis serogroup $\mathrm{B}(\mathrm{NmB})$ (2) due to the low immunogenicity of the serogroup B capsular polysaccharide (3), which is similar to structures found on human neuronal cells (4). In regions where the $\mathrm{A}, \mathrm{C}, \mathrm{Y}, \mathrm{W}$ vaccines are routinely used, endemic $\mathrm{NmB}$ disease is often the most common cause of meningococcal disease in infants, children, and young adults (1). NmB also causes outbreaks in various settings and regions. To control these outbreaks, vaccines based on the highly variable porin A protein were used, but 
these vaccines were not broadly effective against other invasive $\mathrm{NmB}$ strains $(5,6)$.

Recently, two vaccines were developed and licensed for the prevention of $\mathrm{NmB}$ disease: Trumenba (bivalent rLP2086) and Bexsero (4CMenB). While the vaccines have different compositions, they each include factor $\mathrm{H}$ binding protein (fHbp) components (7). fHbp is a surface-expressed lipoprotein capable of inducing serum bactericidal antibodies that can be assessed in serum bactericidal assays that utilize human complement (hSBA). Anti-fHbp antibodies were found in convalescent sera from subjects that contracted meningococcal disease, supporting the expression of fHbps on meningococcal bacteria and their in vivo immunogenicity (8). fHbp is also an important virulence factor expressed by $N$. meningitidis to evade innate immune defenses by specifically binding to complement factor $\mathrm{H}$ (fH) (9). It was suggested that anti-fHbp antibodies can elicit protection by two mechanisms: direct complement-mediated killing of the bacterium and blocking $\mathrm{fH}$ binding to the bacteria to increase the susceptibility of the bacterium to killing by the alternative complement pathway $(7,10)$.

The amino acid sequences of fHbps segregate into two immunologically distinct subfamilies, subfamily A and subfamily B (11). Bexsero contains one recombinant subfamily B protein (v1.1/B24) that is non-lipidated and expressed as a fusion protein (12). B24 is found in $43.8 \%$ and $16.7 \%$ of invasive $\mathrm{NmB}$ strains in the USA and Europe, respectively (13). Thus, additional non-fHbp components were included in Bexsero to extend the coverage of the vaccine to other strains (14). Trumenba contains two lipidated fHbp variants, one from subfamily A (variant A05) and one from subfamily B (variant B01). Early research by Pfizer scientists established that the lipidated version of fHbp elicited broader hSBA responses compared to its non-lipidated form (11).

Bacterial lipoproteins consist of a polypeptide and an $\mathrm{N}$ terminal lipid moiety and are found anchored to the cell surface of bacteria. These lipids commonly contain two fatty acids of various structures acyl-linked to two hydroxyl groups ( $O$-acyl or $O$-linked fatty acid) of a glycerol, which is thioether-linked to the N-terminal cysteine of the mature polypeptide, resulting in a di- $O$-acylated lipid. An additional fatty acid is acyl-linked to the $\alpha$-amine of the $\mathrm{N}$-terminal cysteine ( $N$-acyl or $N$-linked fatty acid), giving rise to a triacylated lipoprotein. A schematic structure of the tri-acylated lipid moiety, containing three C16:0 (16-carbon chain with all saturated bonds) fatty acids, is shown in Fig. 1. Anchoring of the lipid motif in the outer membrane of the bacteria exposes the polypeptide to the bacterial cell surface, thus making it accessible to bactericidal antibodies directed to the protein (15).

In this work, we report the detailed characterization of the structures of rLP2086-A05 and rLP2086-B01, as well as the structure-function relationships, using multiple biochemical and biophysical techniques.

\section{MATERIALS AND METHODS}

\section{Materials}

Trumenba is composed of two fHbps. The subfamily A protein, rLP2086-A05, and the subfamily B protein, rLP2086-

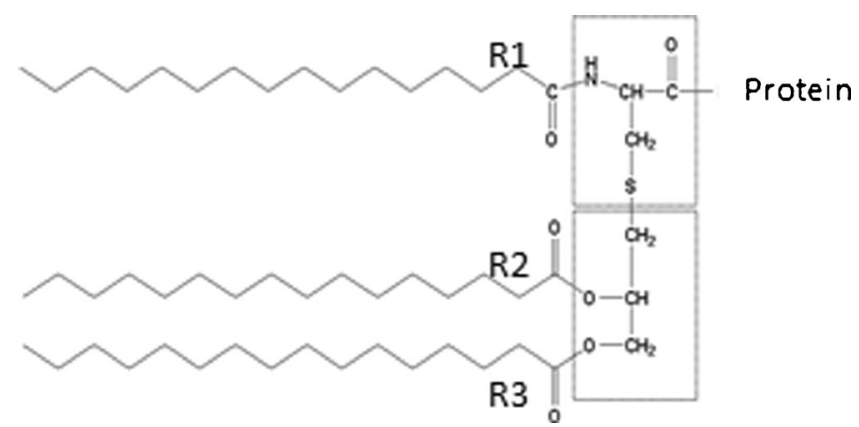

Fig. 1. Schematic structure of a bacterial lipoprotein. The N-terminal $S$-glycerylcysteine is tri-acylated with three palmitoyl fatty acids (C16:0). $R 1$ is the $N$-linked fatty acid; $R 2$ and $R 3$ are the $O$-linked fatty acids

B01, were recombinantly expressed in E. coli suspension cell cultures. The purified bulk solutions of individual lipoproteins, and the vaccine product composed of equal amounts of both lipoproteins, were supplied by Pfizer's manufacturing facility. The non-lipidated version of rLP2086-B01, rP2086B01, was produced as described previously (16). All reagents used were of the highest purity commercially available, and the sources of critical reagents are provided in the methods section.

\section{Methods}

\section{Gas Chromatography-Mass Spectrometry}

Gas chromatography-mass spectrometry (GC/MS) was used to analyze the composition of fatty acids released from rLP2086-A05 and rLP2086-B01 via acid hydrolysis and transesterification to picolinyl esters. The details are described in Supplemental 1.

\section{Peptide Mapping by Liquid Chromatography/Mass Spectrometry}

Non-reduced proteolytic mapping of rLP2086-A05 and rLP2086-B01 with lysyl endoproteinase (Lys-C) was used to characterize the primary sequence and posttranslational modifications of the polypeptide domain, as well as confirming the fatty acid compositions of the lipid moiety. The details are described in Supplemental 1.

\section{Mass of Intact Lipoproteins by LC/MS}

Accurate molecular masses were determined for the intact rLP2086-A05 and rLP2086-B01 by a Waters Acquity UHPLC/UV system interfaced to an ultrahigh-resolution (UHR) Bruker Daltonics maXis electrospray ionization quadrupole time-of-flight (ESI-QTOF) mass spectrometer. The samples were analyzed by injecting approximately $6 \mu \mathrm{g}$ onto a Waters $\mathrm{BEH} \mathrm{C}$ column $(2.1 \times 100 \mathrm{~mm}, 1.7 \mu \mathrm{m})$ at a temperature of $60^{\circ} \mathrm{C}$ and eluted at a flow rate of $0.2 \mathrm{~mL} / \mathrm{min}$ with a gradient from $20 \%$ to $90 \%$ of mobile phase B $(0.1 \%$ TFA in acetonitrile); mobile phase A was $0.1 \%$ TFA in water. The elution profiles were monitored with UV detection at $215 \mathrm{~nm}$. The observed multiply charged ion envelope for each lipidated NmB rLP2086 component was deconvoluted and 
de-isotoped with the Bruker Daltonics maximum entropy and SNAP algorithms, respectively, to generate neutral, monoisotopic masses.

\section{Circular Dichroism and Differential Scanning Calorimetry}

Far-UV circular dichroism (CD) in the 180- to $250-\mathrm{nm}$ range and near-UV $\mathrm{CD}$ in the 250- to 350 -nm range were used to characterize the secondary and tertiary structures of rLP2086 lipoproteins, respectively. Differential scanning calorimetry (DSC) was used to compare their thermal stability. The details are described in Supplemental 1.

\section{Size Exclusion Chromatography and Multi-angle Light Scattering}

Isocratic fractionation by size exclusion highperformance liquid chromatography (SE-HPLC) was performed using Waters Alliance 2695 Separations Module and Tosoh Bioscience TSK G4000SWXL column (7.8 mm $\times$ $30 \mathrm{~cm}, 8 \mu \mathrm{m})$. Other details are described in Supplemental 1.

Raleigh light scattering theory allows determination of the weight average molar mass in a sample if the concentration is known. Therefore, light scattering signals in-line with UV detection provided molar mass information for each SEHPLC fraction. Wyatt Technology DAWN HELEOS II light scattering detector was used. The data were collected and analyzed using the manufacturer-provided software ASTRA v5.3.4.20.

\section{Analytical Ultracentrifugation}

Analytical ultracentrifugation sedimentation velocity experiments were performed on a Beckman XL-I analytical ultracentrifuge to characterize the size distribution of rLP2086s in various solution conditions. Samples were loaded into double-sector (1.2-cm path length) carbonEpon centerpieces in an An-60 Ti rotor. Scans were recorded by UV absorbance at a wavelength of $282 \mathrm{~nm}$ with a spacing of $0.006 \mathrm{~cm}$ in the continuous mode and at $20^{\circ} \mathrm{C}$ using a rotor speed of $42,000 \mathrm{rpm}$ (or as indicated in the figure legend). The sedimentation coefficient distributions, $c(\mathrm{~s})$, were obtained using the program SEDFTT (17).

\section{Lipase De-esterification}

Thermomyces lanuginosus lipase, which is highly specific in hydrolyzing ester-bound ( $O$-linked) fatty acids with no amidase activity (18), was purchased from Sigma (Lipase, L0777) and used to produce de-esterified $N$-acyl rLP2086-A05. The protease impurities were removed using anion exchange chromatography (Q Sepharose) and hydrophobic interaction chromatography (Phenyl Sepharose) prior to the lipid de-esterification treatment to protect the polypeptides of the lipoprotein. Other details are described in Supplemental 1.

\section{In Vitro Toll-Like Receptor Assay Using hTLR2-Transfected Cells}

Commercial HEK-Blue TM hTLR2 cells (InvivoGen \#hkb-htlr2) are produced by co-transfection of the human Toll-like receptor 2 (TLR2), CD14 co-receptor, and SEAP (secreted embryonic alkaline phosphatase) genes into HEK293 cells. The cells also express endogenous levels of TLR1, TLR3, TLR5, and TLR6 (http://www.invivogen.com/ PDF/HEK_Blue_hTLR2_TDS.pdf). Bacterial lipoproteins and lipopeptides are reported to interact only with TLR2 (can be complexed with TLR1 or TLR6), but not with TLR3 and TLR5. Stimulation of the TLR2-transfected cells by TLR ligands can induce the production of SEAP. The phosphatase activity of SEAP, which is proportional to the level of stimulation, can be quantitated using colorimetric assays. HEK293 hTLR2 cells were incubated individually with intact (tri-acylated) NmB rLP2086-A05 vaccine drug substance or drug product, lipase de-esterified $\mathrm{N}$-acyl (mono-acylated) NmB rLP2086-A05, Pam3CSK4 (a commercial TLR2/TLR1 agonist standard; InvivoGen \#tlrl-pms or EMC microcollections \#L2000), or phosphate-buffered saline (PBS; Life Technologies \#10010-023) for 20-22 h at $37^{\circ} \mathrm{C}$. Each sample was made minimally in duplicate. The cell supernatant was diluted into QUANTI-Blue detection medium (InvivoGen \#rep-qb1) and incubated at $37^{\circ} \mathrm{C}$ for $1-3 \mathrm{~h}$ prior to measuring the absorbance at $620-655 \mathrm{~nm}$ for SEAP phosphatase activity. For certain experiments, the cells were pre-incubated with an anti-TLR2 neutralizing monoclonal antibody (InvivoGen \#maba2-htlr2) to discern the role of TLR2 in the resulting response.

\section{Homology Modeling of rLP2086-A05}

A homology model of rLP2086-A05 was built using the nuclear magnetic resonance (NMR) structure of rLP2086-B01 as a template (PDB entry: $2 \mathrm{KDY}$ ). The details are described in Supplemental 1.

\section{RESULTS}

\section{Primary Structure and Posttranslational Modifications}

\section{Identification and Characterization of Fatty Acids by GC/MS}

The E. coli-expressed rLP2086-A05 and rLP2086-B01 are inherently heterogeneous due to expected variations in fatty acid compositions of the lipids, possible posttranslational modifications to amino acids in the polypeptide region, or both. Separate analyses of the fatty acid compositions by $\mathrm{GC} /$ MS and polypeptide structures by liquid chromatographymass spectrometry (LC/MS) provided the basis for complete characterization of the primary structure of both recombinant lipoproteins.

The GC profiles of the picolinyl esters of fatty acids hydrolyzed from the two lipoproteins are shown in Fig. 2. The identifications of fatty acids were based on mass values and the fragmentation patterns. The complete fragmentation achieved for each chromatographically resolved fatty acid also allowed localization of the unsaturated or cyclic groups in the fatty acids. Exemplary MS spectra for C16:0, C16:1, 


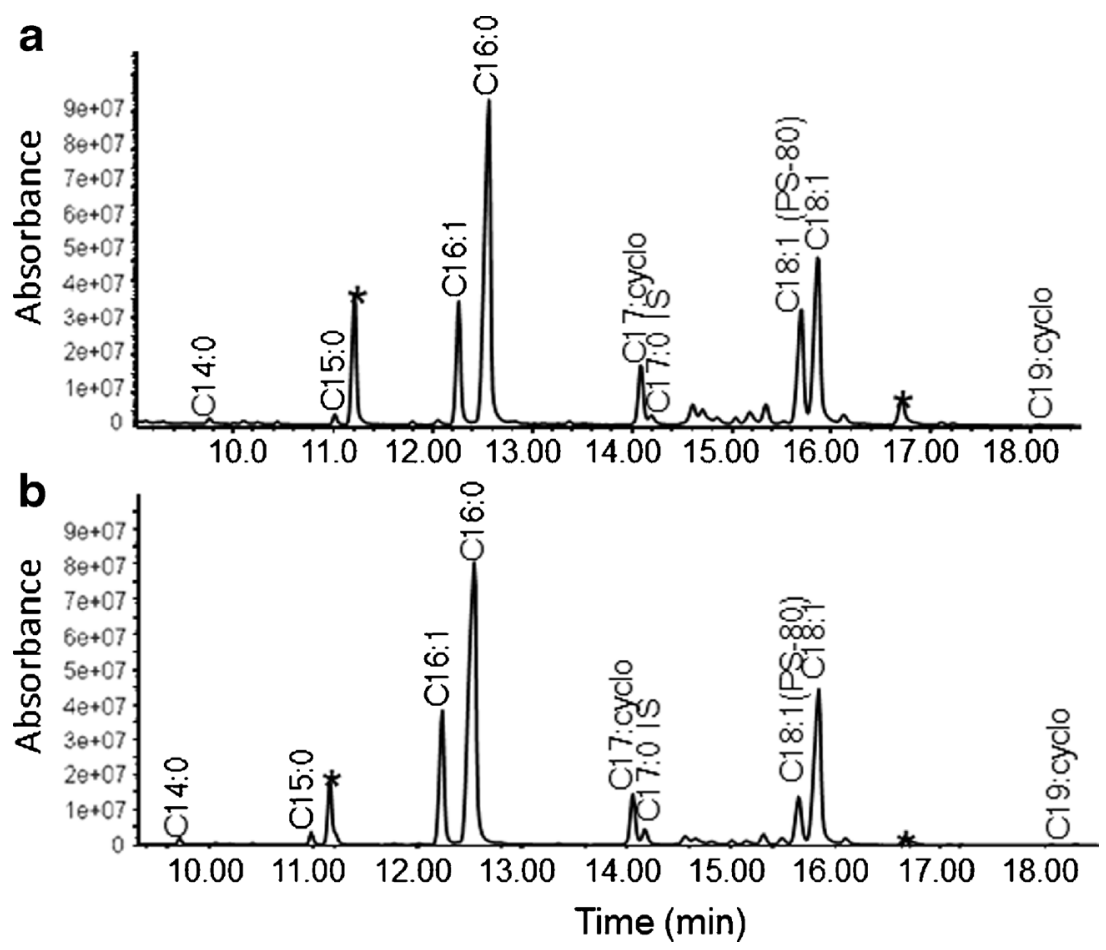

Fig. 2. GC profiles of fatty acid picolinyl esters derived from NmB rLP2086-A05 (a) and rLP2086-B01 (b) lipoproteins. C17:0 IS denotes C17:0 fatty acid that was used as an internal standard. Asterisk denotes system peaks. Peak labels denote assigned fatty acids identified based on molecular mass and fragmentation patterns (see Supplemental 2 Fig. S-1 for exemplary mass spectra). $C$ represents carbon; the first value following $C$ denotes the number of carbons in the fatty acid alkyl chain and the last number represents the number of unsaturated bonds. cyclo denotes a trimethylene cyclic structure. In Trumenba, C16:1 and C17:cyclo are 9,10-hexadecenoic acid and 9,10-methylene hexadecanoic acid, respectively. C18:1 and C19:cyclo are 11,12-octadecenoic acid and 11,12-methylene octadecanoic acid, respectively

and C17:cyclo are shown in Supplemental 2 Fig. S-1. More details for the GC/MS-based structural assignment are described in Supplemental 1.

Despite the structural heterogeneity of the fatty acids in rLP2086-A05 and rLP2086-B01 as assessed by GC/MS, the two lipoproteins exhibited highly similar fatty acid compositions. The three major components-C16:0, C16:1, and C18:1 - accounted for $>90 \%$ of the total fatty acids. The fatty acids at minor and trace levels are C14:0, C15:0, C17:cyclo, and C19:cyclo. These results were confirmed by the LC/MS analyses, as described below. It is noted that no fatty acid shorter than $\mathrm{C} 14$ was observed, which has profound implication to the functionality of the lipid moiety.

\section{Amino Acid Sequence Confirmation and N-Terminal Lipid Structure Elucidation by Peptide Mapping LC/MS}

NmB rLP2086-A05 and rLP2086-B01 are predicted from the DNA sequences to consist of 258 and 261 amino acids, respectively. The two polypeptides share only $\sim 69 \%$ sequence identity. Non-reduced Lys-C peptide mapping via $\mathrm{LC} /$ MS was carried out, as described in "Methods," to analyze the amino acid sequence and posttranslational modifications, as well as to elucidate the compositions of the tri-acyl $\mathrm{N}$ terminal lipid moiety. There are 27 and 22 Lys-C peptides in rLP2086-A05 and rLP2086-B01, respectively, predicted from the respective amino acid sequence. The $\mathrm{N}$-terminal peptides are predicted to contain 28 and 33 amino acids, respectively, each covalently linked to a tri-acylated lipid moiety.

All predicted Lys-C digested amino acid peptides of rLP2086-A05 (L2-L26) and rLP2086-B01 (L2-L21) were identified by mass spectrometry in the peptide region $(<30 \mathrm{~min})$ of the reversed-phase ultrahigh-performance liquid chromatography (RP-UHPLC) peptide map UV profile, as shown in Fig. 3 (L27 of rLP2086-A05 and L22 of rLP2086-B01 are single amino acid residues, $\mathrm{Q}$, at the C-termini, which were not detected). The relative mass errors between the predicted and the observed mass values for all peptides were less than $5 \mathrm{ppm}$. The mass values of all predicted and detected peptides, including the modified peptides, are provided in Supplemental 3 (Tables S-I and S-II).

Due to the hydrophobic nature of the lipids, the $\mathrm{N}$ terminal lipopeptides eluted late in a distinct region ( $>46 \mathrm{~min}$; Fig. 3), which permitted separate analyses of the N-terminal lipopeptides, L1, from all other peptides. This RP-UHPLC method was also capable of resolving most of the lipid isoforms of the N-terminal lipopeptides, as shown in the insets of Fig. 3, which facilitated the elucidation of the fatty acid compositions for the tri-acylated lipid moiety based on the fatty acid structures and their relative abundance attained by GC/MS. The observed monoisotopic molecular masses for 
a
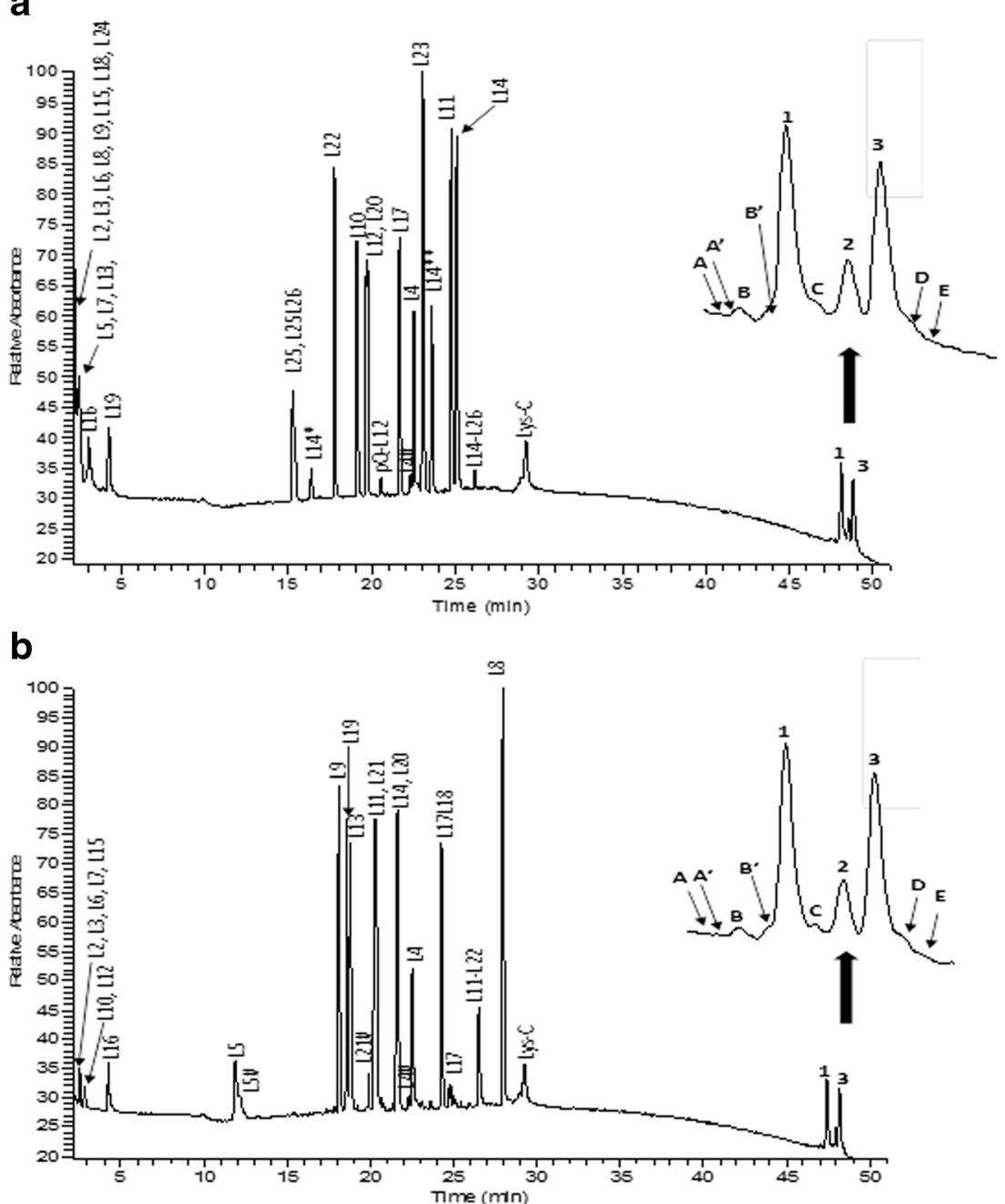

Fig. 3. Lys-C peptide mapping for NmB rLP2086-A05 (a) and rLP2086-B01 (b). Proteolytic peptides were resolved by RP-HPLC and detected using UV and UHR mass spectrometry. Peak labels indicate peptide identifications based on accurate mass determinations (i.e., agreement between the predicted and observed masses within $5 \mathrm{ppm})$. Peaks labeled as $L y s-C$ represent autodigestion peptide products. Other minor level peaks include deamidation (number symbol), pyro-glutamic acid formation (Pyro-Q), and clipping (single and double asterisks). Insets are expanded views of the late-eluting lipidated N-terminal peptides. The structures of the fatty acids in major peaks 1-3 and trace to minor peaks $A-E$ are listed in Tables I and II

the resolved N-terminal lipopeptides are listed in Tables I and II, alongside with the masses predicted from the known amino acids and identified fatty acids via their picolinyl esters. The relative mass errors between the predicted and the observed mass values for all species were less than $5 \mathrm{ppm}$, supporting the high confidence in the lipid structure identification.

\section{LC/MS of Intact NmB Lipoproteins and Characterization of the N-Linked Fatty Acid}

The full-length isoforms of the tri-acylated rLP2086-A05 and rLP2086-B01 lipoproteins can be resolved chromatographically using RP-UHPLC coupled to UHR MS. The profiles, shown in Fig. 4, are similar to those of the N-terminal lipopeptides resolved by the peptide mapping chromatography method. The predicted mass value for a given lipid isoform is based on the amino acid sequence and the N-terminal lipid structure, both confirmed by peptide mapping LC/MS and $\mathrm{GC} /$ MS. The comparisons of the predicted and observed mass values for all observed lipid isoforms for both lipoproteins are listed in Tables III and IV, respectively. The relative mass errors between the predicted and the observed mass values for all species were less than 2 ppm. Greater than 97\% of rLP2086-A05 and rLP2086-B01 molecules are lipidated. Only low levels of degradation products, with partial or no lipidation, were detected.

Further characterization of the $N$-acylated fatty acids was facilitated by de- $O$-acylation using a lipase that specifically removes $O$-linked fatty acids. The lipase-treated rLP2086A05 and rLP2086-B01 were analyzed using the same RPUHPLC/UHR-ESI-QTOF MS method. The lipase digestion 
Table I. Predicted and Observed Masses of NmB rLP2086-A05 N-Terminal Lys-C Peptide Containing Heterogeneous Lipid Isoforms

\begin{tabular}{lllll}
\hline Peak $^{a}$ & Fatty acid composition & & Observed mass $^{d}(\mathrm{Da})$ & Abundance $^{\mathrm{e}}$ \\
\hline A & C16:0, C16:1, C14:0 & Predicted mass & (Da) & Trace \\
A $^{\prime}$ & C16:0, C16:1, C16:1 & 3268.8858 & 3268.8868 & Trace \\
B & C16:0, C16:1, C15:0 & 3294.9014 & 3294.9024 & Minor \\
B $^{\prime}$ & C16:0, C14:0, C16:0 & 3282.9014 & 3282.9007 & Trace \\
1 & C16:0, C16:1, C16:0 & 3270.9014 & 3270.9018 & Major \\
C & C16:0, C16:1, C18:1 & 3296.9171 & 3296.9168 & Minor \\
2 & C16:0, C17:cyclo, C16:0 & 3322.9327 & 3322.9322 & Major \\
3 & C16:0, C18:1, C16:0 & 3310.9327 & 3310.9318 & Major \\
D & C16:0, C18:1, C18:1 & 3324.9484 & 3324.9461 & Minor \\
E & C16:0, C19:cyclo, C16:0 & 3350.9640 & 3350.9623 & Trace \\
\hline
\end{tabular}

${ }^{a}$ Resolved and denoted in the inset of Fig. 3 (top panel)

${ }^{b}$ The order of the fatty acid does not represent the positions of the fatty acids in the tri-acyl lipid moiety

${ }^{c}$ The predicted masses (monoisotopic) for the lipopeptides were calculated based on the masses of the N-terminal 28 amino acids, CGSSGGGGVAADIGTGLADALTAPLDHK, the $S$-glycerol linker, and the predicted fatty acids, in a structure as depicted in Fig. 1

${ }^{d}$ The observed masses (monoisotopic) were derived from the mass spectra following de-isotoping with Extract software (Thermo Scientific)

${ }^{e}$ The abundances were derived from the UV profile

resulted in complete removal of the $O$-linked fatty acids of rLP2086, as demonstrated by the degeneration of the heterogeneous peaks in the 26- to 32-min region in Fig. 4 to a single peak at 13-14 min (Supplemental 2 Fig. S-2). The observed monoisotopic molecular masses of this single peak for these proteins correspond to the masses predicted for the respective polypeptide with an $N$-acylated C16:0 fatty acid on the N-terminus of the thioglycerol-modified cysteine.

\section{Secondary and Tertiary Structures}

\section{Atomistic Structural Models by NMR and Molecular Modeling}

The NMR structure (16) for the non-lipidated NmB fHbp (rP2086) subfamily B, a Cys1Met mutant (11), revealed that the polypeptide folds into $18 \beta$-strands and two short $\alpha$ helices, forming two distinct domains, the $\beta$-sheet $N$-terminal domain and the $\beta$-barrel C-terminal domain. Each domain contains a hydrophobic core formed with hydrophobic side chains of the surrounding amino acids. The hydrophobic core of each domain is protected by positively and negatively charged residues forming large patches on the surface.

An atomic structure for rLP2086-A05 is not available in the Protein Data Bank (PDB) (www.rcsb.org) so far. However, it is expected that the overall higher-order structure of the subfamily A and B proteins, rLP2086-A05 and rLP2086-B01, would be similar since the two molecules share approximately $69 \%$ sequence identity. This expectation of similar higher-order structures is supported by the homology model of rLP2086-A05 built using the NMR structure of rLP2086-B01. Figure S-3 shows the homology model of rLP2086-A05 (orange ribbons) superposed onto the NMR structure of rP2086-B01 (magenta ribbons).

\section{Structural Characterization by Circular Dichroism and Calorimetry}

CD measures differential absorption of the left- and the right-circularly polarized light due to the structural

Table II. Predicted and Observed Masses of NmB rLP2086-B01 N-Terminal Lys-C Peptide Containing Heterogeneous Lipid Isoforms

\begin{tabular}{llll}
\hline Peak $^{a}$ & Fatty acid composition & & Observed mass $^{b}(\mathrm{Da})$ \\
\hline A & C16:0, C16:1, C14:0 & Predicted mass $^{c}(\mathrm{Da})$ & 3614.0186 \\
A $^{\prime}$ & C16:0, C16:1, C16:1 & 3614.0142 & 3640.0316 \\
B & C16:0, C16:1, C15:0 & 3640.0299 & 3628.0340 \\
B & C16:0, C14:0, C16:0 & 3628.0299 & 3616.0348 \\
1 & C16:0, C16:1, C16:0 & 3616.0299 & 3642.0470 \\
C & C16:0, C16:1, C18:1 & 3642.0455 & 3668.0680 \\
2 & C16:0, C17:cyclo, C16:0 & 3668.0612 & 3656.0655 \\
3 & C16:0, C18:1, C16:0 & 3656.0612 & 3670.0812 \\
D & C16:0, C18:1, C18:1 & 3670.0868 & Trace \\
E & C16:0, C19:cyclo, C16:0 & 3696.0925 & Minor \\
\hline
\end{tabular}

${ }^{a}$ Resolved and denoted in the inset of Fig. 3 (bottom panel)

${ }^{b}$ The order of the fatty acid does not represent the positions of the fatty acids in the tri-acyl lipid moiety

${ }^{c}$ The predicted masses (monoisotopic) for the lipopeptides were calculated based on the masses of the N-terminal 33 amino acids, CGSSGGGGSGGGGVTADIGTGLADALTAPLDHK, the $S$-glycerol linker, and the predicted fatty acids, in a structure as depicted in Fig. 1 ${ }^{d}$ The observed masses (monoisotopic) were derived from the mass spectra following de-isotoping with Extract software (Thermo Scientific)

${ }^{e}$ The abundances were derived from the UV profile 
a

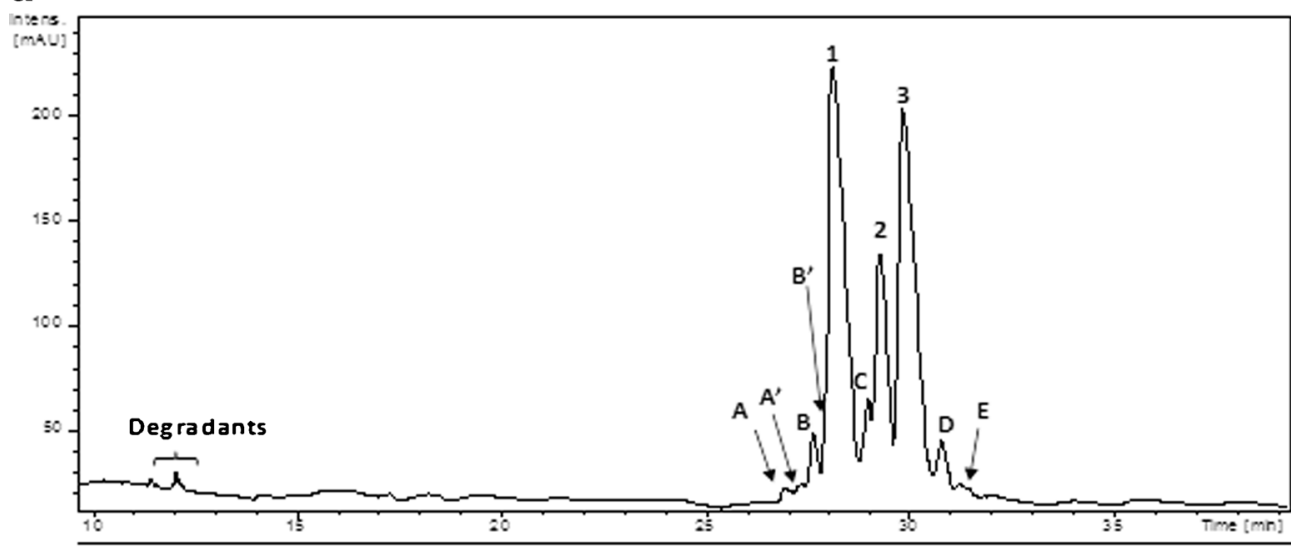

b

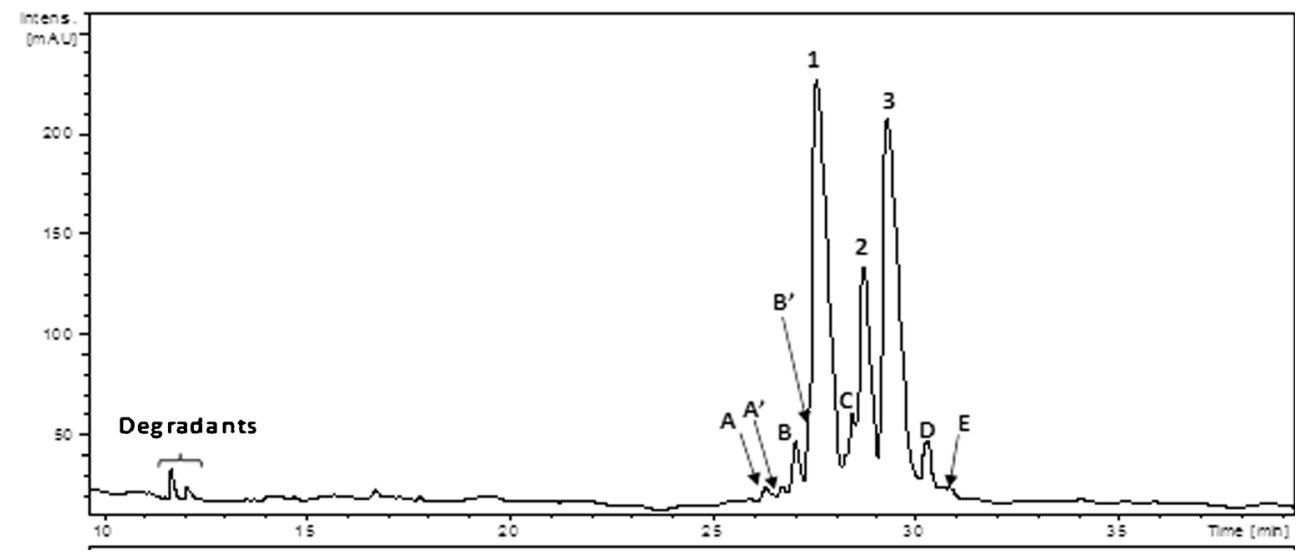

Fig. 4. RP-UHPLC profiles for intact NmB rLP2086-A05 (a) and rLP2086-B01 (b). Peaks between 26 and $32 \mathrm{~min}$ represent isoforms of each $\mathrm{NmB}$ rLP2086 lipoprotein due to the heterogeneous N-terminal lipid compositions. The structures of the fatty acids in major peaks $1-3$ and trace to minor peaks $A-E$ are listed in Tables III and IV. The trace level degradation products eluted around $12 \mathrm{~min}$ are mainly de- $O$-acylated species

asymmetry of molecules. The far-UV CD spectrum for attributable to the amide bonds in polypeptide chains, proteins in the wavelength range of $195-250 \mathrm{~nm}$ is mostly therefore reflecting the secondary structure in protein folding.

Table III. Predicted and Observed Masses of NmB rLP2086-A05 Lipoprotein Containing Heterogeneous Lipid Isoforms

\begin{tabular}{|c|c|c|c|c|c|}
\hline \multirow[t]{2}{*}{$\operatorname{Peak}^{a}$} & \multicolumn{2}{|c|}{ Fatty acid composition } & \multirow[t]{2}{*}{ Predicted $\operatorname{mass}^{c}(\mathrm{Da})$} & \multirow[t]{2}{*}{ Observed mass ${ }^{d}(\mathrm{Da})$} & \multirow[t]{2}{*}{ Abundance } \\
\hline & $N$-linked & $O$-linked ${ }^{b}$ & & & \\
\hline A & C16:0 & $\mathrm{C} 16: 1, \mathrm{C} 14: 0$ & $28,150.725$ & $28,150.764$ & Trace \\
\hline$A^{\prime}$ & $\mathrm{C} 16: 0$ & $\mathrm{C} 16: 1, \mathrm{C} 16: 1$ & $28,176.740$ & $28,176.764$ & Trace \\
\hline B & C16:0 & C16:1, C15:0 & $28,164.740$ & $28,164.769$ & Minor \\
\hline $\mathrm{B}^{\prime}$ & C16:0 & $\mathrm{C} 14: 0, \mathrm{C} 16: 0$ & $28,152.740$ & $28,152.770$ & Trace \\
\hline 1 & $\mathrm{C} 16: 0$ & $\mathrm{C} 16: 1, \mathrm{C} 16: 0$ & $28,178.756$ & $28,178.774$ & Major \\
\hline $\mathrm{C}$ & $\mathrm{C} 16: 0$ & $\mathrm{C} 16: 1, \mathrm{C} 18: 1$ & $28,204.772$ & $28,204.783$ & Minor \\
\hline 2 & $\mathrm{C} 16: 0$ & C17:cyclo, C16:0 & $28,192.772$ & $28,192.779$ & Major \\
\hline 3 & $\mathrm{C} 16: 0$ & $\mathrm{C} 18: 1, \mathrm{C} 16: 0$ & $28,206.787$ & $28,206.777$ & Major \\
\hline $\mathrm{D}$ & $\mathrm{C} 16: 0$ & C18:1, C18:1 & $28,232.803$ & $28,232.810$ & Minor \\
\hline $\mathrm{E}$ & $\mathrm{C} 16: 0$ & C19:cyclo, C16:0 & $28,220.803$ & $28,220.779$ & Trace \\
\hline
\end{tabular}

${ }^{a}$ Resolved and denoted in the inset of Fig. 3 (top panel) and Fig. 4 (top panel)

${ }^{b}$ The order does not represent the positions of the $O$-linked fatty acids

${ }^{c}$ The predicted masses (monoisotopic) for the lipopeptides were calculated based on the masses of the amino acids in rLP2086-A05 sequence, the $S$-glycerol linker, and the predicted fatty acid compositions

${ }^{d}$ The observed masses (monoisotopic) were derived from the mass spectra following deconvolution of the charge envelope with maximum entropy software and de-isotoping with SNAP software (Bruker Daltonics)

${ }^{e}$ The abundances were derived from the UV profile 
Table IV. Predicted and Observed Masses of NmB rLP2086-B01 Lipoprotein Containing Heterogeneous Lipid Isoforms

\begin{tabular}{|c|c|c|c|c|c|}
\hline \multirow[t]{2}{*}{$\operatorname{Peak}^{a}$} & \multicolumn{2}{|c|}{ Fatty acid composition } & \multirow[t]{2}{*}{ Predicted mass ${ }^{c}(\mathrm{Da})$} & \multirow[t]{2}{*}{ Observed mass $^{d}(\mathrm{Da})$} & \multirow[t]{2}{*}{ Abundance $^{\mathrm{e}}$} \\
\hline & $N$-linked & $O$-linked ${ }^{b}$ & & & \\
\hline A & $\mathrm{C} 16: 0$ & C16:1, C14:0 & $28,362.514$ & $28,362.571$ & Trace \\
\hline$A^{\prime}$ & $\mathrm{C} 16: 0$ & C16:1, C16:1 & $28,388.530$ & $28,388.544$ & Trace \\
\hline B & $\mathrm{C} 16: 0$ & $\mathrm{C} 16: 1, \mathrm{C} 15: 0$ & $28,376.530$ & $28,376.572$ & Minor \\
\hline $\mathrm{B}^{\prime}$ & $\mathrm{C} 16: 0$ & $\mathrm{C} 14: 0, \mathrm{C} 16: 0$ & $28,364.530$ & $28,364.527$ & Trace \\
\hline 1 & $\mathrm{C} 16: 0$ & C16:1, C16:0 & $28,390.546$ & $28,390.588$ & Major \\
\hline $\mathrm{C}$ & $\mathrm{C} 16: 0$ & C16:1, C18:1 & $28,416.561$ & $28,416.593$ & Minor \\
\hline 2 & $\mathrm{C} 16: 0$ & C17:cyclo, C16:0 & $28,404.561$ & $28,404.586$ & Major \\
\hline 3 & C16:0 & $\mathrm{C} 18: 1, \mathrm{C} 16: 0$ & $28,418.577$ & $28,418.579$ & Major \\
\hline $\mathrm{D}$ & $\mathrm{C} 16: 0$ & C18:1, C18:1 & $28,444.592$ & $28,444.584$ & Minor \\
\hline E & $\mathrm{C} 16: 0$ & C19:cyclo, C16:0 & $28,432.592$ & $28,432.577$ & Trace \\
\hline
\end{tabular}

${ }^{a}$ Resolved and denoted in the inset of Fig. 3 (bottom panel) and Fig. 4 (bottom panel)

${ }^{b}$ The order does not represent the positions of the $O$-linked fatty acids

${ }^{c}$ The predicted masses (monoisotopic) for the lipopeptides were calculated based on the masses of the amino acids in rLP2086-B01 sequence, the $S$-glycerol linker, and the predicted fatty acid compositions

${ }^{d}$ The observed masses (monoisotopic) were derived from the mass spectra following deconvolution of the charge envelope with maximum entropy software and de-isotoping with SNAP software (Bruker Daltonics)

${ }^{e}$ The abundances were derived from the UV profile

The near-UV CD spectrum for proteins in the wavelength range from 250 to $350 \mathrm{~nm}$ is dominated by the absorbance of aromatic amino acids (Trp and Tyr) and disulfide bonds. It is sensitive to the tertiary folding and quaternary packing of protein polypeptide chains.

As shown in Supplemental 2 Fig. S-4, the far-UV CD spectra for the two proteins exhibit similar features, but are not identical, possibly due to the differences in their amino acid sequences. The near-UV CD spectra are also similar after being normalized against the respective Tyr in each molecule (the two molecules do not contain Trp and disulfide bonds). All CD spectra in Fig. S-4 reflect wellfolded protein polypeptide chains under the conditions that the antigens are known to self-associate and form micellelike complexes due to the hydrophobicity of the N-terminal lipids (see "Quaternary Structure" below).

DSC is commonly used to characterize thermally induced unfolding of proteins by measuring heat capacity as a function of temperature. Figure S-5 in Supplemental 2 shows the thermograms of rLP2086-A05 and rLP2086-B01. Two distinct transitions were observed for rLP2086-B01, whereas rLP2086-A05 exhibited one transition at a temperature slightly higher than the second transition of rLP2086B01.

The thermal unfolding profiles of rLP2086-B01 were previously studied using recombinant polypeptides that correspond to the full-length and the C-terminal domain of the non-lipidated rP2086-B01 (16). The thermogram for the recombinant $\mathrm{C}$-terminal domain is almost identical to the second peak at $85^{\circ} \mathrm{C}$ in the thermogram for the fulllength molecule, suggesting that the second peak is due to the unfolding of the C-terminal domain. Unlike the CD spectra, which largely display the similarity between the two lipoproteins in their secondary folding and tertiary packing, the DSC profiles highlight that some aspect of the higher-order structure is different between these two lipoproteins.

\section{Quaternary Structure}

The size distributions of rLP2086 in non-denaturing conditions, measured by size exclusion chromatography inline with multi-angle light scattering detectors (SEC-MALS), are shown in Fig. 5a, b. The measured weight average molar mass is $\sim 668,000 \mathrm{~g} / \mathrm{mol}$ for rLP2086-A05 and $\sim 660,000 \mathrm{~g} / \mathrm{mol}$ for rLP2086-B01. Based on the monomeric molar masses of both molecules being $\sim 28,000 \mathrm{~g} / \mathrm{mol}$, these measured average masses suggest that fHbp lipoproteins associate into complexes of approximately 24 mers.

The large complexes of rLP2086-A05 and rLP2086B01 are likely to exist as micelles. Both proteins contain highly hydrophobic tri-acylated lipids at the N-termini and high charge density on the surface of the folded polypeptide domains (16). They are, therefore, highly amphiphilic. On the bacterial cell surface, the lipids anchor the lipoproteins to bacterial cell walls by embedding in the membrane bilayer. In the purified vaccine solutions, which are free of membrane components, it is energetically favorable for the lipids of multiple molecules to associate with each other and form a hydrophobic core of a micelle, with the hydrophilic polypeptide domains on the outer layer to assemble (self-associate) in a side-by-side manner, as illustrated in Fig. 5c.

To confirm that the micellar structure is driven by the association of the hydrophobic lipids, the size distribution of the non-lipidated polypeptide of rP2086-B01 was analyzed using analytical ultracentrifugation sedimentation velocity (AUC-SV), as shown in Supplemental 2 Fig. S-6. The measured size was independent of the protein concentration, with the peak sedimentation coefficient corresponding to the monomeric rP2086 molecule. This result supports that the lipids of fHbp lipoproteins are causing the formation of the micelles, and the micellar state is the natural quaternary structure of the lipoproteins in the vaccine formulation. 

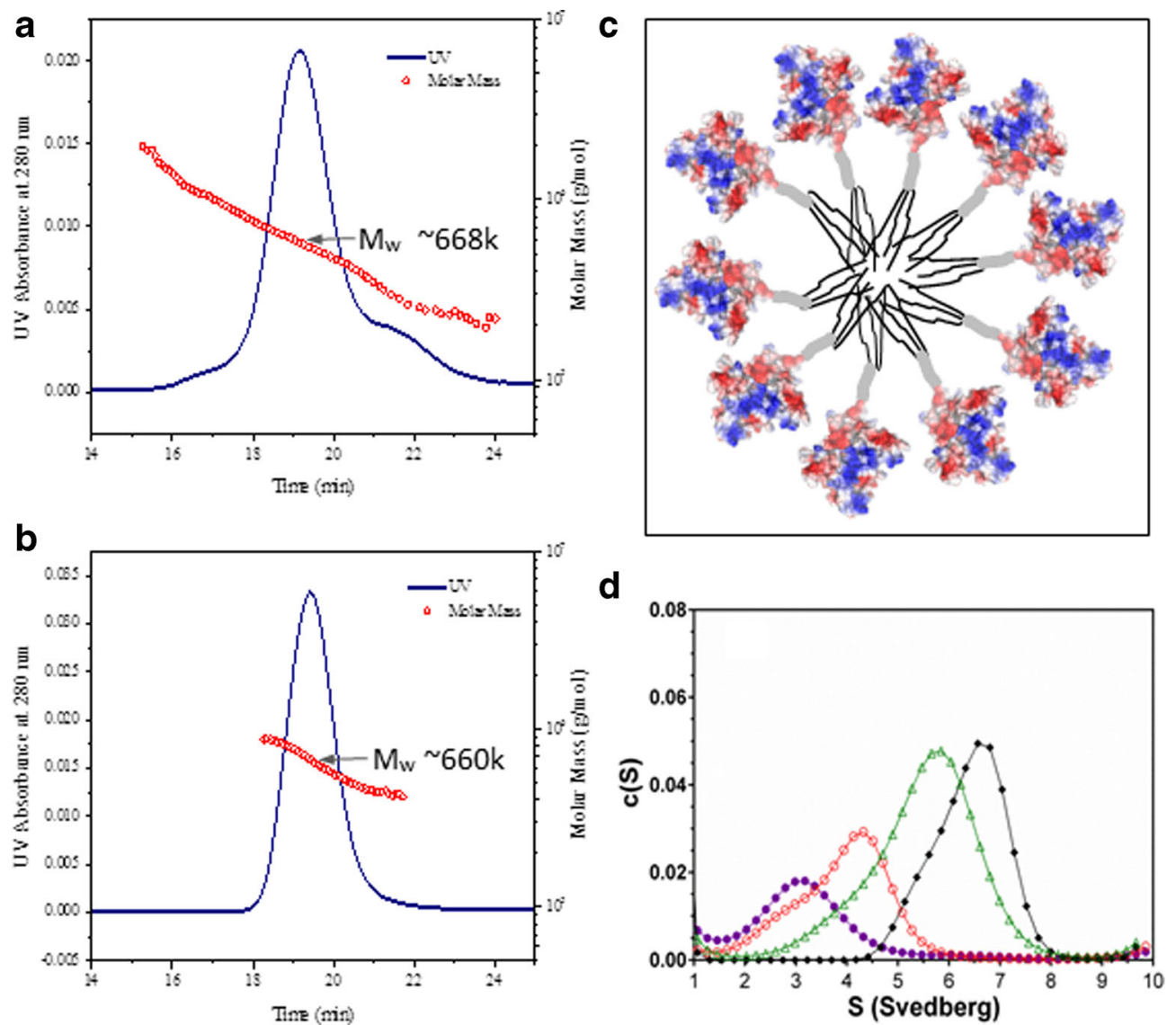

Fig. 5. SEC-MALS measurements for NmB rLP2086-A05 (a) and rLP2086-B01 (b). The chromatograms by UV detector (blue lines) are superimposed with the molar mass values (red circles) obtained by MALS detector in-line with the UV detector. c Schematic model of micelles in NmB rLP2086 lipoprotein vaccines. The bulky and charged polypeptide chains and the elongated and hydrophobic lipid moiety form the outer layer and the core of the micelles, respectively. The tertiary structure of the polypeptide chain is based on the NMR structure for NmB rLP2086-B01. d Sedimentation coefficient distributions by AUC for NmB rLP2086-B01 at $3.6 \mu \mathrm{M}$ (purple line and dot), $5.5 \mu \mathrm{M}$ (red line and circle), $8 \mu \mathrm{M}$ (black line and diamond), and $10 \mu \mathrm{M}$ (green line and triangle) in PBS with $0.1 \%$ Zwittergent 3-10

Chemical denaturation was performed to explore possible roles of the protein polypeptide structure in micelle formation. In the presence of $8 \mathrm{M}$ urea in the SEC mobile phase, the hydrodynamic size of rLP2086-A05 micelles appears to be increased (Supplemental 2 Fig. S-7). This result suggests, firstly, that the micelles were not dismantled by the protein denaturant. The increase of the micellar size upon the unfolding of the polypeptide domains can be due to the more extended and flexible polypeptide chains of each molecule in the micelles, which may increase the effective hydrodynamic radius. Secondly, as the polypeptide domains become less bulky and rigid, space is made available for more molecules, leading to the increased average mass of the micelles.

The above results indicate that the optimal number of molecules per micelle is determined by the length of the lipid moiety relative to the size/volume of the polypeptide domain, with longer lipids being in favor of larger micelles and larger or bulkier polypeptide domains being in favor of smaller micelles by limiting the number of molecules in a micelle. This implies that the size range of micelles formed by a given lipoprotein should not be concentration- dependent at concentrations above the critical micellar concentration (CMC). The SEC profiles shown in Fig. 5a, b are largely independent of the loading concentration when greater than $0.2 \mathrm{mg} / \mathrm{mL}(\sim 7 \mu \mathrm{M})$. Below CMC, micelles are in concentration-dependent dynamic equilibria with nonassociated monomers. AUC-SV was used to study the size distribution below $0.2 \mathrm{mg} / \mathrm{mL}$. Figure $5 \mathrm{~d}$ shows the $c(\mathrm{~s})$ profiles for rLP2086-B01 solutions from $3.5 \mu \mathrm{M}(\sim 0.1 \mathrm{mg} /$ $\mathrm{mL})$ to $10 \mu \mathrm{M}(\sim 0.28 \mathrm{mg} / \mathrm{mL})$. At $3.5 \mu \mathrm{M}$, the average size was approaching that of non-lipidated molecules (see Supplemental 2 Fig. S-6), while at concentrations above $8 \mu \mathrm{M}$, the average size reached a maximum. Therefore, the estimated CMC for $\mathrm{rLP} 2086$ is $\sim 0.2 \mathrm{mg} / \mathrm{mL}(\sim 7 \mu \mathrm{M}$, or $\sim 0.02 \% \mathrm{w} / \mathrm{v}$ ) at $20^{\circ} \mathrm{C}$. No surfactant other than Zwittergent 3-10 at a low level $(0.1 \%$, lower than the CMC) was present in the solution.

The formulation of Trumenba contains low level of polysorbate 80 (PS-80), which is commonly used to increase the stability of biologics. The micelle-prone lipoproteins and surfactant molecules constitute a binary micellar system. The characterization of such a system consisting of rLP2086 and PS-80 is described in Supplemental 1. 
Functional Role of the N-Terminal Lipids

Interactions of $\mathrm{NmB}$ Lipoproteins with hTLR2-Expressing Cells

The immune-enhancing effect of the N-terminal lipids of rLP2086s was demonstrated previously by comparing the results of the lipidated and non-lipidated recombinant $\mathrm{fHbp}$ molecules in the serum bactericidal antibody assay, which measures the capability of the antibodies in the sera of immunized mice to kill target meningococcal strains in a complement-dependent fashion $(11,19)$. The mechanism for the immune-enhancing effect studied with other bacterial lipopeptides and lipoproteins suggests lipoprotein recognition of TLR receptors on cells of the innate immune system (20-23). In this study, the interactions of rLP2086 with TLR2 and/or TLR2/TLR1 complexes on cell surfaces, and the subsequent cell activation, were confirmed using HEK293 hTLR2 cells that express human TLR2 and TLR1. Upon TLR2 activation, the cells express secreted embryonic alkaline phosphatase (SEAP), which can be quantitated. In this assay, the tri-acylated lipopeptide Pam3CSK4, which is a standard TLR2/TLR1 agonist, and PBS were used as the positive and negative controls, respectively. The Trumenba sample, containing equal amounts of rLP2086-A05 and rLP2086-B01 lipoproteins, stimulated HEK293 hTLR2 cells in a dose-dependent manner similar to the activity observed with Pam3CSK4 (Fig. 6a).

\section{Critical Role of TLR2 in Cell Activation by NmB Lipoproteins}

The central role of TLR2 in the activation of HEK293 hTLR 2 cells by Trumenba antigens was further confirmed by inhibiting cell activation with a neutralizing anti-TLR2 antibody. The cells were incubated with anti-TLR2 monoclonal antibody (mAb) at various concentrations prior to the stimulation by Pam3CSK4 or the NmB rLP2086 components at fixed concentrations. The cell activations by both agonists increased as anti-TLR2 mAb decreased (Fig. 6b), suggesting that the cellular responses to the agonists require specific interactions of these agonists with TLR2 on the cell surface.

Critical Role of the O-Linked Fatty Acids in the Activation of TLR2-Expressing Cells

The critical role of the $O$-linked fatty acids was further demonstrated by comparing the activation of HEK-Blue hTLR2 cells induced by the intact and the lipase-treated rLP2086-A05, as shown in Fig. 6c. Lipase treatment resulted in the removal of the two $O$-linked fatty acids, giving rise to the mono- $N$-acyl NmB rLP2086-A05, or de- $O$-acylated $\mathrm{NmB}$ rLP2086-A05. The purified material contained full-length protein polypeptide and the $N$-linked fatty acid, predominantly C16:0 (Supplemental 2 Fig. S-2 and Tables III and IV). Lipopeptide Pam3CSK4 was also used as a comparator. At similar molar concentrations, the intact NmB rLP2086-A05 exhibited a high cell activation activity close to that of Pam3CSK4, while the lipase-treated mono- $N$-acyl $\mathrm{NmB}$ rLP2086-A05 exhibited significantly attenuated activity.
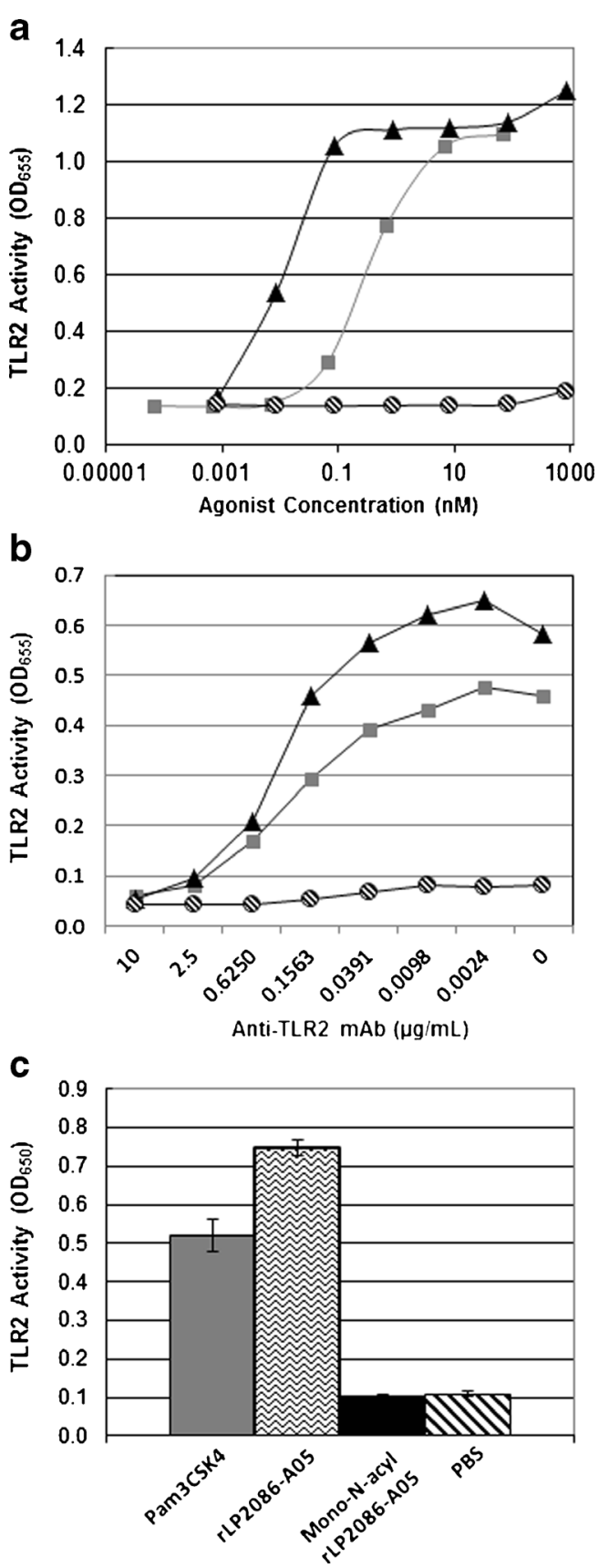

Fig. 6. a Activation of HEK 293 hTLR2 cells by Pam3CSK4 standard (solid gray square) and Trumenba vaccine (solid black triangle) containing equal amounts of NmB rLP208-A05 and rLP2086-B01 lipoproteins. PBS solution (thatched circle) was used as the negative control. Each data point represents the average of duplicate tests. b Effect of anti-TLR2 mAb on the activation of HEK293 hTLR2 cells induced by PAM3CSK4 (0.67 nM, solid gray square) and Trumenba vaccine $(0.08 \mathrm{nM}$, solid black triangle) containing equal amounts of $\mathrm{NmB}$ rLP208-A05 and rLP2086-B01. PBS solution (thatched circle) was used as the negative control. Each data point represents the average of duplicate tests. c Activation of HEK293 hTLR2 cells by Pam3CSK4 (solid gray), NmB rLP2086-A05 (zigzag), and mono- $N$ acyl rLP2086-A05 (solid black). PBS solution (thatched) was used as the negative control. The activities of the lipopeptide and lipoprotein samples were compared at a fixed concentration of $0.66 \mathrm{nM}$ in the assay. Bar graphs represent the mean and error bars represent the standard deviation of $N=14$ replicates. The $Y$-axis for all panels is in absorbance units at the indicated wavelengths 
Taken together, the results described above support the perspective that the presence of the $O$-linked fatty acids in the tri-acylated NmB rLP2086 lipoproteins is critical for the stimulation of TLR2/TLR1-expressing cells and, thus, explain the immune enhancement observed with the lipidated forms of fHbps compared to their non-lipidated forms.

\section{DISCUSSION}

The Polypeptide and Lipid Domains of Trumenba Lipoproteins Play Distinct Structural Roles

\section{The Polypeptide Domain of rLP2086}

The atomic structure of NmB rLP2086-B01 polypeptide by NMR in the solution phase was consistent with the X-ray crystal structures reported for similar $\mathrm{NmB}$ lipoproteins $(24,25)$. Both showed that the polypeptides of the lipoproteins fold into mainly two conformational motifs in two sideby-side domains, i.e., the $\mathrm{N}$-terminal and the C-terminal domains. This domain structure was shown to be important for factor $\mathrm{H}$ binding to fHbps (24), which allows meningococcal bacteria to avoid the human innate immune system. The human factor $\mathrm{H}(\mathrm{hfH})$ in the crystal structure is shown to simultaneously interact with both the N-terminal and the Cterminal domains. The atomic structure for NmB rLP2086A05 has not been resolved. Given the fact that the two fHbp subfamilies share similar hfH binding property, it is likely that the conformations of the $\mathrm{hfH}$ binding sites in the two subfamilies are similar. The hfH binding sites contain large numbers of subfamily-defining residues $(25,26)$. The regions that are largely composed of conserved residues are expected to be also similar. This is supported by the superimposed crystal structures of a v3 variant (corresponds to one branch of subfamily A (14)) with a v1 variant (corresponds to subfamily B (14)) (25) and by the homology model built for rLP2086-A05 using the NMR structure of rLP2086-B01 (Supplemental 2 Fig. S-3). The far-UV and near-UV CD spectra, which reflect the secondary folding and tertiary packing of protein polypeptides, respectively, of $\mathrm{NmB}$ rLP2086-A05 and rLP2086-B01 are similar to each other at all wavelengths (Supplemental 2 Fig. S-4). This indicates that, in the solution phase, the overall structural features of the two molecules in the native state are similar.

\section{The Lipid Motif of rLP2086}

One of the novel findings in this work is the micellar quaternary structure of NmB rLP2086-A05 and rLP2086-B01 lipoproteins, as well as the binary micellar systems in the presence of surfactant molecules, via size distribution analyses using AUC and SEC-MALS. Structurally modified materials, such as non-lipidated proteins, were used to demonstrate the role of the lipids in the formation of lipoprotein micelles (Supplemental 2 Fig. S-6). A denaturing condition for proteins was applied to examine the impact of the spatial dimensions of the polypeptide domain on the size distribution of the lipoprotein micelles (Supplemental 2 Fig. S-7). It was concluded that the hydrophobicity of the lipid moiety in the lipoproteins drives the self-association of the purified lipoproteins to form micelles. This is similar to the role of anchoring the lipoproteins to the bacterial cell walls by embedding themselves in the lipid bilayer membrane (16). The average size of the micelles is determined by the optimal number of lipoproteins that fit in individual micelles. This is, in turn, determined by the relative dimensions of the lipids and the polypeptide region. Under the conditions used in the SEC-MALS studies (Fig. 5a, b), this number is on average $\sim 24$ for purified Trumenba components. Significant changes in the structure of the lipids or conformation of the protein polypeptides can induce changes in the size distribution of the micelles.

\section{Immune-Enhancing Effects of the N-Terminal Lipids of rLP2086}

During the design of Trumenba, it was noted in preclinical in vivo studies that the lipidated forms of fHbps were more immunogenic compared to the non-lipidated forms; thus, the lipidated forms were chosen as vaccine antigens (11). To fully elucidate the lipid structures of the Trumenba antigens and to understand the immune-enhancing mechanism, the lipidated structures were thoroughly characterized by chromatographic techniques and mass spectrometry. Data shown in Figs. 3 and 4, for the N-terminal peptides and the intact molecules, respectively, demonstrated that $>97 \%$ of rLP2086-A05 and rLP2086-B01 are lipidated, and all lipid isoforms are tri-acylated with fatty acids of 14 carbons or longer (Tables I, II, III, and IV), in agreement with fatty acids detected by GC/MS. The characterization data for Trumenba reported in this work are consistent with data in the literature for other E. coli-produced lipoproteins, e.g., rAg473 (27).

When associated with the bacteria, the lipids of the bacterial lipoproteins play mainly a structural role of anchoring the lipoproteins to bacterial cell walls. However, during infection, the fHbp lipoproteins can dissociate from the bacteria and the exposed lipid moiety can play different roles in the disease-causing infection. It was discovered in the late 1980s that conjugation of Pam3CSS (tripalmitoyl Cys-Ser$\mathrm{Ser}$ ) or Pam2CSS (di- $O$-acylated palmitoyl Cys-Ser-Ser) to peptides derived from bacterial or viral proteins could convert the immunologically inactive peptides into potent vaccines that provided long-lasting protection against bacterial or viral infections in animal models. This effect was enabled by induction of neutralizing antibodies $(28,29)$ and by in vivo priming of epitope-specific cytotoxic $\mathrm{T}$ cells and memory T cells (30-32). Since antibodies to the lipid moieties are not generated in vivo, the immune-enhancing effect of the lipids is believed to be similar to that of a conventional adjuvant, and thus, bacterial lipoprotein/lipopeptidecontaining vaccines are recognized as one category of selfadjuvanting vaccines (21-23,33). Other categories include lipid-core peptides, single-chain palmitoylated peptides, and glycolipid analogues of bacterial lipopolysaccharides (21).

Complete understanding of the mechanism for the adjuvanting activity of various lipids attached to polypeptide immunogens is still evolving. It was perceived in early days that the lipids simply anchor the lipoproteins/lipopeptides to the surface membrane of immune cells to facilitate the internalization. Recent research suggested that the lipid moieties interact with various TLRs, a type of membranebound pattern recognition receptors (PRRs), to drive the 
endocytosis of the lipoproteins by antigen-presenting cells (34) and induce various immune responses $(21,22,32)$. PRR is a key component of the innate immune system to identify pathogen-associated molecular patterns (PAMPs) (35).

More than ten human TLRs have been identified so far that are involved in recognizing different PAMPs. TLR2 is essential in the recognition of bacterial lipoproteins/ lipopeptides (20,36). TLR2 in combination with other TLRs determine the specificity for lipid structures. Crystal structures of the TLR2-TLR1 complex interacting with a triacylated lipopeptide, Pam3CSK4, and the TLR2-TLR6 complex interacting with a di- $O$-acylated lipopeptide, Pam2CSK4, were solved (37). The two $O$-linked fatty acids in both structures are shown to be in parallel in the lipidbinding channel of TLR2, while the $N$-linked fatty acid of Pam3CSK4 is in the lipid-binding channel of TLR1. It was suggested that the simultaneous interactions of the triacylated lipid with the ectodomains of TLR2 and TLR1, or the di-acylated lipids with TLR2 and TLR6, bring the Cterminal intracellular domains of these receptors into a closer contact (36).

In human blood, TLR2 has the highest expression on CD14 ${ }^{+}$monocytes (38), which mature and differentiate into phagocytes such as dendritic cells (DC) and macrophages when migrating to tissues. In DC, the dimerization of the intracellular domains of TLR2 and TLR1 or TLR6 triggers a cascade of intracellular signal transductions and kinase activations that result in the de-inhibition of $\mathrm{NFkB}$, a nuclear factor that controls the transcription of DNA. The free NFkB can translocate to the nucleus, where it binds to the enhancer or promoter regions of target genes and regulates their transcription (22). The target genes of NFkB control all the functions associated with DC maturation, and thus the chain of events leading to the priming of the immune responses, which likely include (1) activation of antigen-specific $\mathrm{CD} 4^{+} \mathrm{T}$ cells that can secrete cytokines and induce B cells to produce antigen-specific antibodies against the internalized, processed, and presented peptide epitopes; (2) activation of cytotoxic $\mathrm{CD}^{+} \mathrm{T}$ cell that can lyse infected cells and differentiate to long-lived memory T cells; and (3) secretion of cytokines that activate other immune cells, e.g., NK cells and phagocytes, to attack the infected cells (22). The killing of pathogens and infected cells by cytotoxic T cells and NK cells can also be induced by direct interactions of bacterial lipoproteins with TLR2 on the surface of these immune cells in the absence of TLR1 (39,40). However, only the activated B cells can produce functional antibodies and can differentiate to memory $\mathrm{B}$ cells that produce antigen-specific antibodies upon future invasion of the same bacteria, therefore eliminating these bacteria more rapidly. By this mechanism, the epitopecontaining polypeptide domain of the lipoproteins functions as the antigen, with the attached lipids functioning as adjuvants that prime the immune system for stronger responses to the antigens. Together, they constitute selfadjuvanting vaccines.

The two lipoproteins in Trumenba, rLP2086-A05 and rLP2086-B01, which contain identical lipid isoforms (Tables I, II, III, and IV), exhibited characteristics of the abovedescribed self-adjuvanting vaccines and, as shown previously, were required for enhanced immune responses compared to the non-lipidated forms (11). Further, the de- $O$-acylated molecules were shown in this work to be inactive by an in vitro assay using hTLR2-expressing HEK293 cells (Fig. 6c). The critical role of TLR2 in cell stimulation by Trumenba was shown by specific blocking of TLR2 with anti-TLR2 antibodies (Fig. 6b). These results are consistent with the reports that the $O$-linked fatty acids only interact with TLR2 (36) and that TLR2 is critical in the activation of the immune system by bacterial lipoproteins (22). We therefore conclude that the Trumenba antigens constitute a self-adjuvanting vaccine, with the protein polypeptides giving rise to functional bactericidal antibodies, and the N-terminal lipids serving as an adjuvant, capable of activating the innate immune system via the recognition by TLR2 and promoting its crosstalk with the adaptive immune system to produce antibodies against the polypeptide epitopes.

However, the critical role of the lipids in interacting with TLR2 appears to conflict with the role of the lipids in lipoprotein micelles in the Trumenba formulation, which also increase the stability of the purified lipoprotein molecules. The former requires the lipid moiety to be fully accessible, while the latter results in the burial of the lipids in the hydrophobic core of the micelles. If the lipid moiety remains buried in the micelles following vaccination, the interactions with TLRs may be less effective, hence a lower immuneenhancing effect. To achieve both the desired stability before vaccination and a high immunogenic efficacy after vaccination, the lipoproteins need to form micelles in the formulation and then dissociate upon administration. This requires formulating the lipoproteins at concentrations slightly above the $\mathrm{CMC}$ of the lipoprotein micelles, which is estimated for $\mathrm{NmB}$ rLP2086 lipoproteins to be $\sim 0.2 \mathrm{mg} / \mathrm{mL}$. Each dose of Trumenba contains a total of $0.12 \mathrm{mg}$ lipoproteins $(0.06 \mathrm{mg}$ rLP2086-A05 and $0.06 \mathrm{mg}$ rLP2086-B01) formulated in $0.5 \mathrm{~mL}$ solution, resulting in a total concentration of $0.24 \mathrm{mg} / \mathrm{mL}$. When the vaccine diffuses following the administration and reaches in vivo concentrations below $0.2 \mathrm{mg} / \mathrm{mL}$, the micelles will disperse, exposing the lipid moiety of individual lipoproteins to interact with their target TLRs.

\section{All Lipid Isoforms in Trumenba Are Effective Adjuvants—Effects of Lipid Structures on TLR2-Dependent Stimulation of Immune Responses}

The crystal structures of the TLR2-TLR1 complex interacting with tri-acylated lipids, and of the TLR2-TLR6 complex interacting with di- $O$-acylated lipids, provided structural basis for the specificity of surface receptors of the innate immune cells in recognizing lipidated bacterial components, but are not sufficient to determine the impact of finer structures of the fatty acids in the lipids on the potency to stimulate immune responses. Extensive experimental studies were conducted to address these gaps for the purpose of developing more potent lipopeptide vaccines, taking advantage of modern technologies to synthesize lipopeptides and the stable cell lines that express desired human receptors.

Synthetic tri-acyl-CSSNASK4 lipopeptides with the $\mathrm{N}$ linked fatty acids being all Pam $(\mathrm{C} 16: 0)$ and the two $O$-linked fatty acids varying from $\mathrm{C} 6$ to $\mathrm{C} 20$, some containing unsaturated bonds (Ole and Lin are both $\mathrm{C} 18$, Ole contains one unsaturated bond (C18:1), and Lin contains two 
unsaturated bonds (C18:2)), were studied using HEK293 hTLR2 cell lines (41). The results by monitoring IL-8 secretion and NFkB activation showed similar dependence of cell activation on structures of the $O$-linked fatty acids. Both assays observed baseline-level activities, with the carbon chains of the $O$-linked fatty acids ranging from C6 to C10. There were then sharp increases in the activity with the $O$ linked fatty acids from $\mathrm{C} 10$ to $\mathrm{C} 14$. From C14 to C20, IL-8 secretion was more or less plateaued, whereas NFkB activation continued to increase.

Other factors, including the length of the $N$-linked fatty acids, were also investigated in the above-mentioned work. The observed structural requirements for tri-acylated lipopeptides to exhibit full immune activation activity are: (1) The minimum length of the two $O$-linked fatty acids is $\mathrm{C} 14$; (2) lipids with both $O$-linked fatty acids from $\mathrm{C} 14$ to $\mathrm{C} 20$ exhibit similar activities; (3) the minimum length of the $\mathrm{N}$ linked fatty acid for full activity is C6; (4) lipids with the $\mathrm{N}$ linked fatty acids from C6 to C18 exhibit similar activities; and (5) the $O$-linked fatty acids $\geq \mathrm{C} 14$ with one or two unsaturated bonds exhibit similar activities to the corresponding saturated fatty acids.

The two recombinant bacterial lipoproteins in Trumenba, rLP2086-A05 and rLP2086-B01, exhibit heterogeneities that are resolvable by RP-HPLC (Fig. 4). The components under the peaks eluted between 26 and $32 \mathrm{~min}$ have been identified by chromatographic and mass spectrometry techniques to be lipid variants (Tables I, II, III, and IV). Within each subfamily, no major difference in polypeptide chain was observed; all differences in mass were due to the heterogeneity in the lipid structure. The two lipoproteins contained the same lipid variants with similar distributions. Further, the lipid variants, which were predominantly triacylated, contained the same $N$-linked fatty acid, Pam (C16:0), as well as the two $O$-linked fatty acids, which differentiate the lipid variants via combinations of fatty acids of lengths ranging from $\mathrm{C} 14$ to $\mathrm{C} 19$, with saturated, unsaturated, and cyclic structures. The key finding is that all the lipid isoforms in each subfamily meet the above-described structural requirements, i.e., fatty acids $\geq \mathrm{C} 14$, for full immune stimulation activity. Therefore, all lipidated molecules in Trumenba should have similar self-adjuvanting activities.

\section{CONCLUSIONS}

Trumenba is a well-characterized vaccine composed of two recombinant bacterial lipoproteins, NmB rLP2086-A05 and rLP2086-B01, which are tri-acylated with fatty acids of 14-19 carbon atoms in length. Both lipoproteins self-associate and exhibit a micelle-like quaternary structure due to the hydrophobicity of the N-terminal lipids, which enhances stability of the product $(\mathrm{CMC} \sim 0.2 \mathrm{mg} / \mathrm{mL}$ or $\sim 7 \mu \mathrm{M})$. The $\mathrm{N}$-terminal lipid motif was previously indicated to be critical to the immune enhancement (11). It is demonstrated in this work that the lipids interact with the TLR2/TLR1 complex, which stimulates cell activation. Further, the two $O$-linked fatty acids are responsible for all the activation effect via their exclusive interactions with TLR2. It is, therefore, concluded that Trumenba is a self-adjuvanting vaccine, with the lipid motif of the lipoprotein components playing the role of adjuvant. It is the first example of a licensed vaccine that has a documented adjuvant activity incorporated into its target antigens. One of the novelties of Trumenba is the dual role of the N-terminal lipids in stabilizing the higher-order structure of rLP2086 and in the self-adjuvanticity of the vaccine.

\section{ACKNOWLEDGMENTS}

Sincere thanks and appreciation go to Margaret Ruesch, Mark Ruppen, Marta Czupryn, David Cooper, Thomas Warren, Marja van Zeijl, and the entire Pfizer BioTherapeutics Pharmaceutical Sciences and Vaccine Research and Development organizations for their support, guidance, and critical review. The following former and current Pfizer employees are acknowledged for their individual contributions to the study. David S. Snyder is thanked for the fatty acid identifications via GC/MS. Daniel Haq, Cliff Entrican, Sharon Polleck, and Tanya Shang, Suddham Singh, and Ksenia Krylova are thanked for their technical contributions. Lucas Wafer, Marek Kloczewiak, Paul Czajak, Len Blackwell, Michele Bailey Piatchek, and Qin Zou are thanked for helpful scientific discussions and manuscript review. The study was sponsored by Pfizer Inc.

\section{COMPLIANCE WITH ETHICAL STANDARDS}

Conflict of Interest All authors are current or former employees of Pfizer Inc.

Open Access This article is distributed under the terms of the Creative Commons Attribution 4.0 International License (http://creativecommons.org/licenses/by/4.0/), which permits unrestricted use, distribution, and reproduction in any medium, provided you give appropriate credit to the original author(s) and the source, provide a link to the Creative Commons license, and indicate if changes were made.

\section{REFERENCES}

1. Sridhar S, Greenwood B, Head C, Plotkin SA, Sáfadi MA, Saha $\mathrm{S}$, et al. Global incidence of serogroup B invasive meningococcal disease: a systematic review. Lancet Infect Dis. 2015;15:1334-46.

2. Bruge J, Bouveret-Le Cam N, Danve B, Rougon G, Schulz D. Clinical evaluation of a group B meningococcal N-propionylated polysaccharide conjugate vaccine in adult, male volunteers. Vaccine. 2004;22:1087-96.

3. Wyle FA, Artenstein MS, Brandt BL, Tramont EC, Kasper DL, Altieri PL. Immunologic response of man to group B meningococcal polysaccharide vaccines. J Infect Dis. 1972;126:514-21.

4. Finne J, Leinonen M, Makela PH. Antigenic similarities between brain components and bacteria causing meningitis. Implications for vaccine development and pathogenesis. Lancet. 1983;2:355-7.

5. Holst J, Martin D, Arnold R, Huergo CC, Oster P, O'Hallahan J, et al. Properties and clinical performance of vaccines containing outer membrane vesicles from Neisseria meningitidis. Vaccine. 2009;27:B3-12.

6. Tappero JW, Lagos R, Maldonado Ballesteros A, Plikaytis B, Williams D, Dykes J, et al. Immunogenicity of 2 serogroup B outer-membrane protein meningococcal vaccines: a randomized controlled trial in Chile. JAMA. 1999;281:1520-7.

7. Seib KL, Scarselli M, Comanducci M, Toneatto D, Masignani V. Neisseria meningitidis factor H-binding protein fHbp: a key virulence factor and vaccine antigen. Expert Rev Vaccines. 2015;14:841-59. 
8. Ala'aldeen DA, Flint M, Oldfield NJ, Omer SA, McNeil LK, Jiang $\mathrm{Q}$, et al. Human antibody responses to the meningococcal factor $\mathrm{H}$ binding protein (LP2086) during invasive disease, colonization and carriage. Vaccine. 2010;28:7667-75.

9. Seib KL, Serruto D, Oriente F, Delany I, Adu-Bobie J, Veggi D, et al. Factor H-binding protein is important for meningococcal survival in human whole blood and serum and in the presence of the antimicrobial peptide LL-37. Infect Immun. 2009;77:292-9.

10. Madico G, Welsch JA, Lewis LA, McNaughton A, Perlman DH, Costello CE, et al. The meningococcal vaccine candidate GNA1870 binds the complement regulatory protein factor $\mathrm{H}$ and enhances serum resistance. J Immunol. 2006;177:501-10.

11. Fletcher LD, Bernfield L, Barniak V, Farley JE, Howell A, Knauf M, et al. Vaccine potential of the Neisseria meningitidis 2086 lipoprotein. Infect Immun. 2004;72:2088-100.

12. Jiang HQ, Hoiseth SK, Harris SL, McNeil LK, Zhu D, Tan C, et al. Broad vaccine coverage predicted for a bivalent recombinant factor $\mathrm{H}$ binding protein based vaccine to prevent serogroup B meningococcal disease. Vaccine. 2010;28:6086-93.

13. Hoiseth SK, Murphy E, Andrew L, Vogel U, Frosch M, Hellenbrand W, et al. A multi-country evaluation of Neisseria meningitidis serogroup B factor H-binding proteins and implications for vaccine coverage in different age groups. Pediatr Infect Dis J. 2013;32:1096-101.

14. McNeil LK, Zagursky RJ, Lin SL, Murphy E, Zlotnick GW, Hoiseth SK, et al. Role of factor H binding protein in Neisseria meningitidis virulence and its potential as a vaccine candidate to broadly protect against meningococcal disease. Microbiol Mol Biol Rev. 2013;77:234-52.

15. Bos MP, Robert V, Tommassen J. Biogenesis of the Gramnegative bacterial outer membrane. Ann Rev Microbiol. 2007;61:191-214.

16. Mascioni A, Bentley BE, Camarda R, Dilts DA, Fink P, Gusarova V, et al. Structural basis for the immunogenic properties of the meningococcal vaccine candidate LP2086. J Biol Chem. 2009;284:8738-46.

17. Schuck P. Size-distribution analysis of macromolecules by sedimentation velocity ultracentrifugation and Lamm equation modeling. Biophys J. 2000;78:1606-19.

18. Fernandez-Lafuente R. Lipase from Thermomyces lanuginosus: uses and prospects as an industrial biocatalyst. J Mol Cat B: Enzymatic. 2010;62:197-212.

19. Seib KL, Brunelli B, Brogioni B, Palumbo E, Bambini S, Muzzi $\mathrm{A}$, et al. Characterisation of diverse sub-variants of the meningococcal factor $\mathrm{H}$ binding protein (fHbp) for their ability to bind $\mathrm{fH}$, mediate serum resistance and induce bactericidal antibodies. Infect Immun. 2011;79:970-81.

20. Takeda K, Takeuchi O, Akira S. Recognition of lipopeptides by Toll-like receptors. J Endotoxin Res. 2002;8:459-63.

21. Moyle PM, Toth I. Self-adjuvanting lipopeptide vaccines. Curr Med Chem. 2008;16:506-16.

22. Brown LE, Jackson DC. Lipid-based self-adjuvanting vaccines. Curr Drug Deliv. 2005;2:383-93.

23. Lex A, Wiesmüller KH, Jung G, Bessler WG. A synthetic analogue of Escherichia coli lipoprotein, tripalmitoyl pentapeptide, constitutes a potent immune adjuvant. J Immunol. 1986;137:2676-81.

24. Schneider MC, Prosser BE, Caesar JJE, Kugelberg E, Li S, Zhang Q, et al. Neisseria meningitidis recruits factor $\mathrm{H}$ using protein mimicry of host carbohydrates. Nature. 2009;458:890-3.
25. Johnson S, Tan L, van der Veen S, Caesar J, Goicoechea De Jorge E, Harding RJ, et al. Design and evaluation of meningococcal vaccines through structure-based modification of host and pathogen molecules. PLOS Pathogens. 2012;8:e1002981.

26. Murphy E, Andrew L, Lee KL, Dilts DA, Nunez L, Fink PS, et al. Sequence diversity of the factor $\mathrm{H}$ binding protein vaccine candidate in epidemiologically relevant strains of serogroup B Neisseria meningitidis. J Infect Dis. 2009;200:379-89.

27. Kwok Y, Sung W-C, Lin AL-H, Liu H-H, Chou FA-H, Hsieh SS$\mathrm{Y}$, et al. Rapid isolation and characterization of bacterial lipopeptides using liquid chromatography and mass spectrometry analysis. Proteomics. 2011;11:2620-7.

28. Jung G, Wiesmuller K-H, Becker G, Buhring H-J, Bessler WG. Increased production of specific antibodies by presentation of the antigen determinants with covalently coupled lipopeptide mitogens. Angew Chem Int Ed Engl. 1985;24:872-3.

29. Wiesmüller K-H, Jung G, Hess G. Novel low-molecularweight synthetic vaccine against foot-and-mouth disease containing a potent B-cell and macrophage activator. Vaccine. 1989;7:29-33.

30. Deres K, Schild H, Wiesmuller K-H, Jung G, Rammensee H-G. In vivo priming of virus-specific cytotoxic $\mathrm{T}$ lymphocytes with synthetic lipopeptide vaccine. Nature. 1989;342:561-4.

31. Brown LE. The role of adjuvants in vaccines for seasonal and pandemic influenza. Vaccine. 2010;28:8043-5.

32. Lahiri A, Das P, Chakravortty D. Engagement of TLR signaling as adjuvant: towards smarter vaccine and beyond. Vaccine. 2008;26:6777-83.

33. Chua BY, Eriksson EM, Brown LE, Zeng W, Gowans EJ, Torresi J, et al. A self-adjuvanting lipopeptide-based vaccine candidate for the treatment of hepatitis $\mathrm{C}$ virus infection. Vaccine. 2008;26:4866-75.

34. Schjetne KW, Thompson KM, Nilsen N, Flo TH, Fleckenstein B, Versen JG, et al. Cutting edge: link between innate and adaptive immunity: Toll-like receptor 2 internalizes antigen for presentation to $\mathrm{CD}^{+} \mathrm{T}$ cells and could be an efficient vaccine target. J Immunol. 2003;171:32-6.

35. Medzhitov R, Janeway CAJ. Innate immunity: the virtues of a nonclonal system of recognition. Cell. 1997;91:295-8.

36. Jin MS, Kim SE, Heo JY, Lee ME, Kim HM, Paik S-G, et al. Crystal structure of the TLR1-TLR2 heterodimer induced by binding of a tri-acylated lipopeptide. Cell. 2007;130:1071-82.

37. Kang JY, Nan X, Jin MS, Youn S-J, Ryu YH, Mah S, et al. Recognition of lipopeptide patterns by Toll-like receptor 2-Tolllike receptor 6 heterodimer. Immunity. 2009;31:873-84.

38. Flo TH, Halaas $\varnothing$, Torp S, Ryan L, Lien E, Dybdahl B, et al. Differential expression of Toll-like receptor 2 in human cells. J Leukoc Biol. 2001;69:474-81.

39. McSharry BP, Gardiner CM. The role of NK cells in bacterial infections. In: Zimmer J, editor. Natural killer cells. Berlin: Springer; 2010. p. 153-75.

40. MacLeod H, Wetzler LM. T cell activation by TLRs: a role for TLRs in the adaptive immune response. Sci STKE. 2007;2007:pe48.

41. Buwitt-Beckmann U, Heine H, Wiesmuller K-H, Jung G, Brock R, Ulmer AJ. Lipopeptide structure determines TLR2 dependent cell activation level. FEBS J. 2005;272:6354-64. 